\title{
A Deep Learning-based Dengue Mosquito Detection Method Using Faster R-CNN and Image Processing Techniques
}

\author{
Rumali Siddiqua ${ }^{1}$, Shakila Rahman ${ }^{2}$ and Jia Uddin ${ }^{3, *}$ \\ ${ }^{1}$ Swinburne University of Technology, Melbourne, Australia \\ rumali.siddiqua@g.bracu.ac.bd \\ 2International Islamic University Chittagong, Chittagong, Bangladesh \\ shakila.rahman1119@gmail.com \\ ${ }^{3}$ Woosong University, Daejeon, South Korea \\ jia.uddin@wsu.ac.kr \\ *Correspondence: jia.uddin@wsu.ac.kr
}

Received: 20 $0^{\text {th }}$ February 2021; Accepted: 21 $1^{\text {st }}$ June 2021; Published: $1^{\text {st }}$ July 2021

\begin{abstract}
Dengue fever, a mosquito-borne disease caused by dengue viruses, is a significant public health concern in many countries especially in the tropical and subtropical regions. In this paper, we introduce a deep learning-based model using Faster R-CNN with InceptionV2 accompanied by image processing techniques to identify the dengue mosquitoes. Performance of the proposed model is evaluated using a custom mosquito dataset built upon varying environments which are collected from the internet. The proposed Faster R-CNN with InceptionV2 model is compared with other two state-of-art models, R-FCN with ResNet 101 and SSD with MobilenetV2. The False positive (FP), False negative (FN), precision and recall are used as performance measurement tools to evaluate the detection accuracy of the proposed model. The experimental results demonstrate that as a classifier the Faster- RCNN model shows $95.19 \%$ of accuracy and outperforms other state-of-the-art models as R-FCN and SSD model show $\mathbf{9 4 . 2 0 \%}$ and $\mathbf{9 2 . 5 5 \%}$ detection accuracy, respectively for the test dataset.
\end{abstract}

Keywords: Deep Learning; Dengue Mosquito; Detection Algorithms; Faster R-CNN; Image Processing; Inception 2

\section{Introduction}

An infectious disease is a terrible threat and causes destruction for a nation. It affects the growth of public health and social sustainability. Mosquito-borne viruses like Malaria, Dengue, West Nile Fever, Zika Fever, etc. are the world's fastest-spreading infectious diseases ${ }^{1}$. Dengue is a viral infection exacerbated by four types of the Flaviviridae family of viruses (DENV-1, DENV-2, DENV3 , and DENV-4). The viruses spread through bites of contaminated female mosquitoes named Aedes aegypti and Aedes albopictus ${ }^{1}$. These mosquitoes are also a vector of Chikungunya, yellow fever, and Zika viruses ${ }^{2}$. The disease has been prevalent in the South Asian countries where there is a heavy rain that provides the ideal breeding ground for the mosquito-borne virus during the monsoon

\footnotetext{
${ }^{1}$ https://www.who.int/news-room/fact-sheets/detail/dengue-and-severe-dengue.

${ }^{2}$ https://www.cdc.gov/dengue/about/index.html.
} 
season. To alleviate the spread of infectious diseases, it is necessary to combat the spread of dengue mosquitoes. Identification method of dengue mosquitoes is complicated as there are more than 3,000 different species of them ${ }^{3}$. Conventional approaches to mosquito control generally focus on adult mosquito control through the thermal convection cooling trucks, insecticides, or even advanced electric mosquito traps [1]. Moreover, trained professionals set traps to drive mosquitoes to a certain area to isolate and collect them. Occasionally, the collected mosquitoes can be trapped within a single day. The specimen is then placed under a microscope to be identified and labelled which is a timeconsuming process. This process can be performed several times in a single week depending on the location and time of year that is cognitively challenging [2]. Furthermore, understanding the different mosquito species can significantly modify trap placement and insecticidal spraying. Different mosquito species are vectors for various diseases and knowing their characteristics can help prioritize immediate attention during the peak times.

However, severe dengue has a greater risk of death if not treated properly. Up to 400 million people get dengue-infected each year. This has devastating effects on public health putting negative impact on the national and global economy. One forecasting estimate shows that 390 million dengue virus infections per year (95\% accurate range 284-528 million), of which 96 million with some seriousness of the disease are medically manifested. The report on dengue prevalence states approximately 3.9 billion individuals are at risk of disease by the dengue virus infection [3]. Based on the facts presented, a modern technology-based solution for identifying dengue mosquitoes from other species that is accurate, effective, and rapid should be developed and deployed. It will also be combined with an increasing awareness of the global spread of dengue mosquito diseases, allowing for more effective use of human resources in public health. The significant research vision is to develop a model that facilitates and focuses on improving prediction of dengue infection. This will enable healthcare experts to create strategy for coping up with the disease. Researchers are now collecting and analysing data to correctly recognize the cognitive factors which drive the spread of the disease. Moreover, they are also developing a variety of predictive modelling methods using statistical and mathematical analysis, and machine learning.

The goal of our research is to study the feasibility of using the deep learning-based object detection methods to identify dengue mosquitoes by their texture characteristics. Nonetheless, in the process of image classification and detection, deep learning approaches have primarily been used. It can function well even if the features are limited along with its ubiquitous ability to comprehend different characteristics that human beings cannot to identify or classify images of different environments [4]. Convolutional Neural Networks (CNN) [5] have become the state-of-the-art in object recognition and classification. It is widely recognized in most detection-based applications. Deep learning-based detection models overwhelm the efficiency of the conventional identification process. Handcrafted features and shallow adaptable architectures are the fundamentals of traditional detection algorithms. By constructing a complex structure that combines various low-level image features with high-level information from object detectors and image classifiers, their output can effectively stabilize. Consequently, modern detection models' network architecture, training strategy, and modelling feature are efficient than the conventional detection techniques [6].

In this paper, three different detection methods: Faster-Region-Based Convolutional Neural Networks (Faster R-CNN) [7], Region-based Fully Convolutional Networks (R-FCN) [8], and Single Shot MultiBox Detector (SSD) [9] with feature extraction network InceptionV2 [10], Resnet 101 [11], and MobilenetV2 [12] are trained, validated and tested to compare the performance. The parameters of the three algorithms are presented in such a way that the detection algorithms are compared according to time and accuracy. We have analyzed the architecture for using these object detection methods to create an effective and reliable framework for detecting dengue mosquitos from images, as well as emerging of technologies that adapt to modern deep learning-based detection algorithms.

\footnotetext{
${ }^{3}$ https://www.nationalgeographic.com/animals/invertebrates/facts/mosquitoes
} 


\section{Literature Review}

Object detection has gained significant attention from researchers in recent years because of its association with image recognition. Image processing methods such as detection based on Histogram of Oriented Gradients (HOG) [13], object detection using Haar-like features [14], and machine learning-based methods such as support vector machine (SVM) [15], artificial neural network (ANN) [16], background removal [17] have been commonly used in traditional image classification or detection. Compared to the conventional machine learning approaches, recent developments in architectures of the deep neural network defined by the CNN have shown a considerable improvement in efficiency $[18,19]$. As the significance of mosquito control has increased, several experiments have been performed to use neural networks so that it can predict mosquitoes from a single image [20,21]. An image-based insect classification system has been proposed in this research [22] using four methods of feature extraction: Hu moments (Hu), Elliptic Fourier Descriptors (EFD), Radial Distance Functions (RDF), and Local Binary Patterns (LBP); but these images require manual preprocessing, which is definitely time intensive. Okayasu et al. [23] implemented a vision-based approach to classify mosquito species. By collecting image datasets of three types of mosquito species, a deep learning approach together with data augmentation have been considered effective for the mosquito species classification with greater accuracy. The aim of this study is to compare traditional classification systems focused on handcraft features and CNN-related deep learning methods. However, the images captured with a smartphone do not show the differences in colour if the shooting environment is dark, because a smartphone camera cannot provide a quality capture compared to reflex single-lens cameras eventually resulting in misclassification.

An automatic mosquito detection platform based on vision, which can function for the inhabitants of closed-perimeter mosquitoes is deployed in [24]. The module would classify mosquitoes extracting morphological features from other species, such as bees and flies, accompanied by the help of support vector machine-based classification. This vision is based on the idea that addressing the problem of mosquito detection throughout this way provides an important alternative to conventional mosquito monitoring, mapping, and sampling methods. The C-SVC SVM module, which has been proposed in this method, has a maximum accuracy of $85.2 \%$ in terms of the proportion of images obtained when detecting mosquitoes. Nonetheless, they should have included additional features in classification efficiency and research extension to compare alternative methods, such as neural networks, genetic algorithms, and in the case of mosquito classification, defining distinguishable characteristics for sub-species classification of mosquitoes.

A solution to detecting Aedes aegypti species using images has been proposed in [25]. They have used a 500x optical zoom sensor, and support vector machine. A confusion matrix has also been used to demonstrate the system's precision in detecting Aedes Aegypti. However, it is still necessary to develop a faster program for detecting mosquito properties through the implementation of other techniques in MATLAB as a part of the framework, since processing the file can take a long time. Moreover, this approach is costly and only solves a binary classification problem. Similarly, another image processing and deep learning method for the bacteria recognition system has been introduced in [26], but the results indicated that standard image resolution datasets for bacteria could be better in the future. In [27], 7 species and 60 mosquitoes have been classified using the random forest algorithm, depending on classifiers and the denoted pixel values. The verification however, has been inadequate since the number of images is around 60. In [28], research on imaging techniques for the classification of insects is presented. However, mosquitoes are not categorized there.

A dengue detection method using cell phone vision sensors with a lightweight object recognition algorithm has been presented in [29]. It has provided an efficient way to use the mobile phone's computing power to detect dengue and a minor medical patch that transforms the various colors of shades. Consequently, in defining input images, the active contouring algorithm was more efficient, but in order to connect an image of the object, the algorithm requires several complex sets of image operations. In [30], the anticipation number of confirmed dengue fever (DF) with three different prediction models based on machine learning and deep learning approach has been applied. Among 
the three different models, the GA-RNN model provides the best performance. However, this work needs to improve the output of the LR model by reducing its appropriate shifting effect.

Current dengue mosquito research usually reflects a set of domains of general detection methods and does not represent the state-of-the-art approaches that include any innovative solutions in the field of deep learning-based detection. This paper analyses deep learning-based dengue mosquito detection approaches in a systematic way. Using the advanced deep learning techniques, we present possible solutions and future research directions in image processing.

\section{Proposed Methodology}

In this section, we represent the implementation procedure of the detection algorithm. This research is aimed at training the $\mathrm{CNN}$ object detection classifier for dengue mosquito detection. The diagram of workflow for complete dengue mosquito detection procedure is presented in Figure 1 which consists of the following blocks: data pre-processing (data acquisition, data annotation), data processing, training and testing the deep classifier.

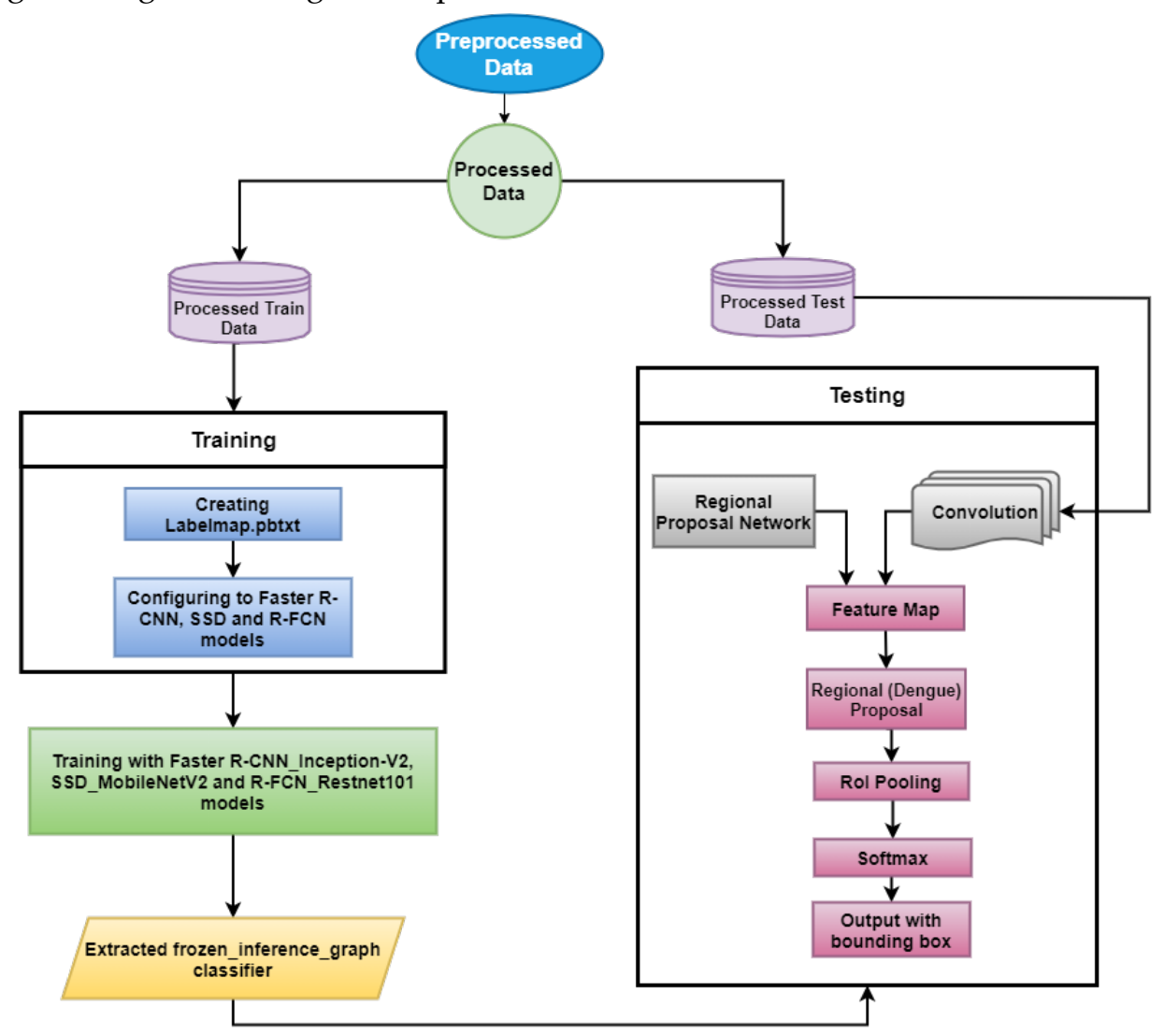

Figure 1. Diagram of the workflow for the whole detection procedure

\subsection{Data Pre-processing}

Initially, data is required to implement the pre-processing task collected in compliance with the desired condition. Detailed data pre-processing procedures are depicted in Figure 2 and described in the next sections. 


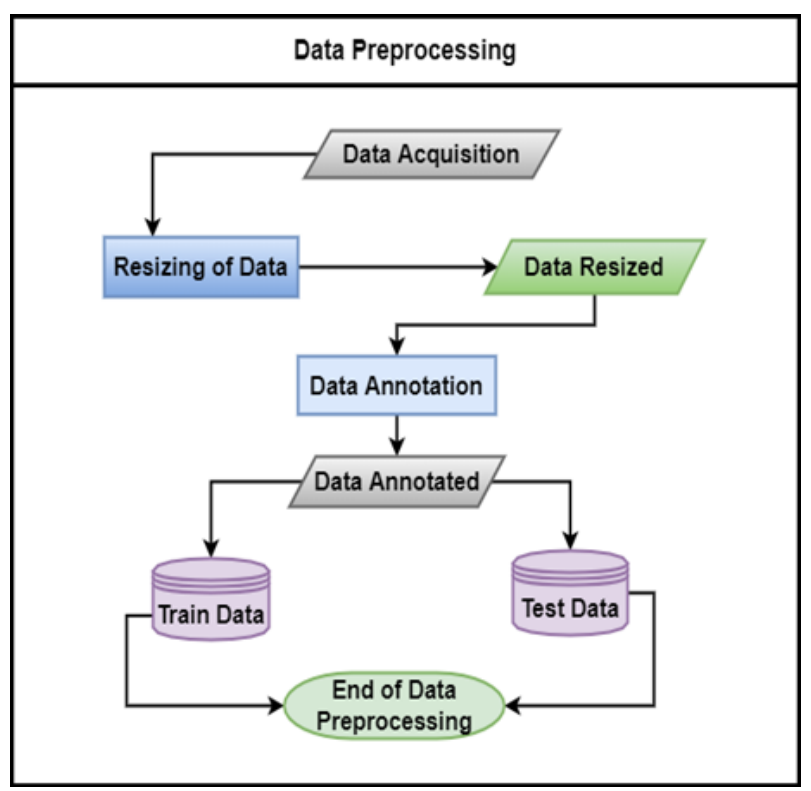

Figure 2. Workflow diagram for Data Pre-processing

\subsubsection{Data Acquisition}

The input image is initially identified as an image of a dengue mosquito or another image of insects (For example, flies, grasshopper, moths). Normal mosquitoes have a slim body with long legs and fairly long antennas with very similar shape and size segments. Dengue mosquitoes have longerlegs than the normal mosquitoes and have white stripes on their legs and bodies. Data has been obtained from the video (transformed into images), self-captured images, and various relevant internet resources under different conditions. As mosquitoes and insects always move, it is difficult to obtain clear images directly while they are flying. A total of 241 dengue mosquito images are collected to form a dataset. Samples of the dataset is shown in Figure 3 and Figure 4. After the data acquisition process, the images have been reformatted to a predefined size of $800^{*} 600$. The resized image involves the conversion into the same size of many of the images. The entire data set is initially distributed into two datasets, the training set, and the test set. The total dataset is divided into $85 \%$ and $15 \%$ for training and testing respectively.
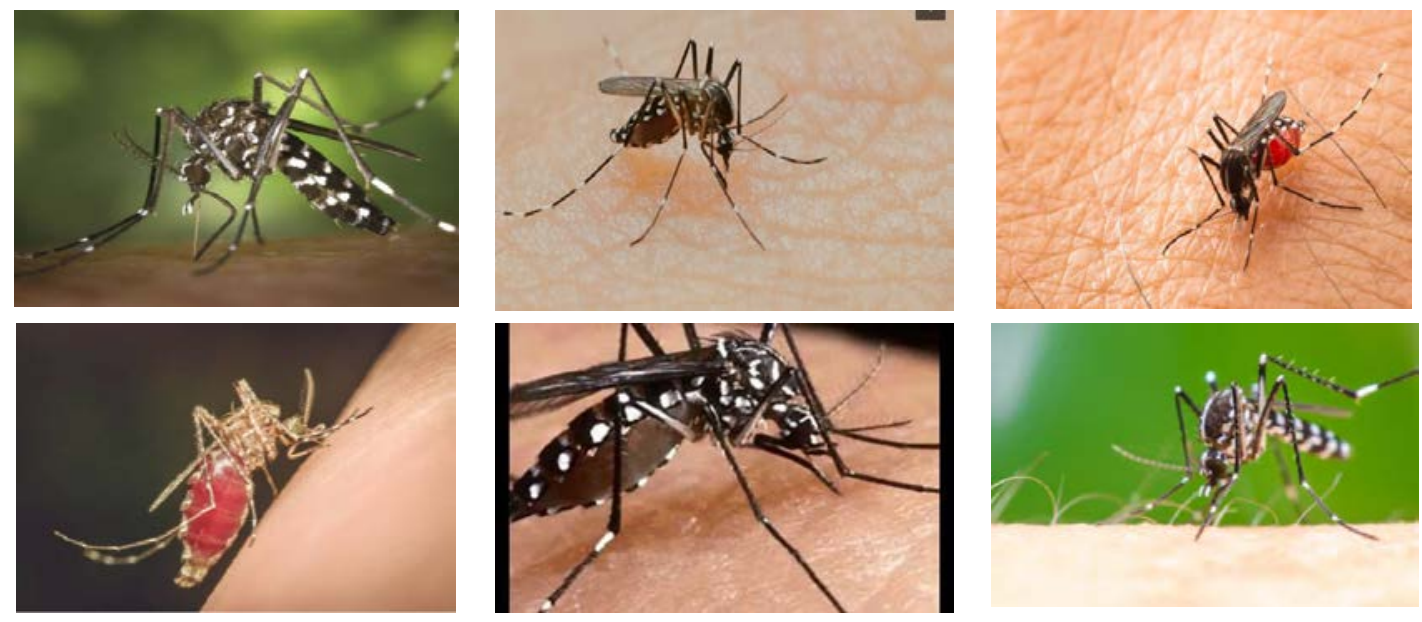

Figure 3. Some sample collected images of dengue mosquitoes
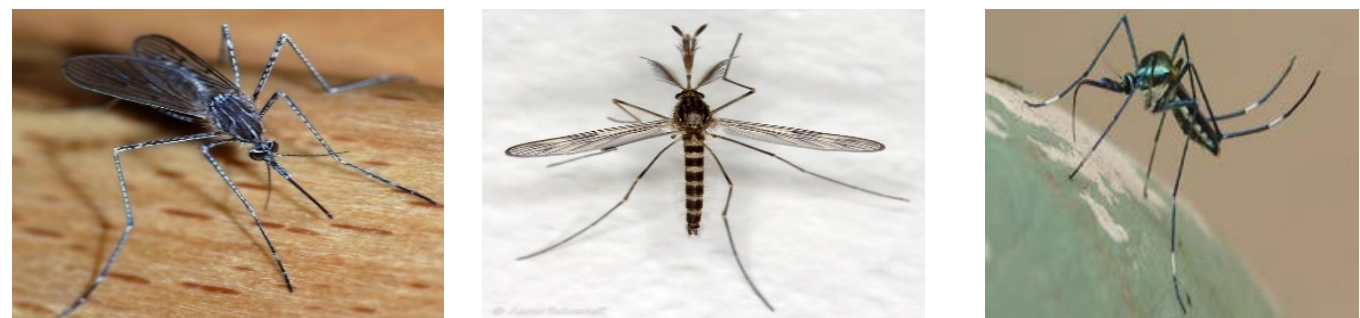

Figure 4. Some sample collected images of insects 


\subsubsection{Data Annotation}

This is a method of identifying the appropriate data in different formats, such as text, video, or images. After collecting the dengue mosquitoes' images from the different environmental conditions, the desired image is selected using the labeling software. It draws visual boxes in the image around a dengue mosquito and saves the XML files for that image containing the label data for each image automatically. The dengue mosquito has been identified according to the region of interest (ROI) and labeled 'dengue mosquito' in the image for accurate detection. Figure 5 shows the annotated portion of the sample image data.
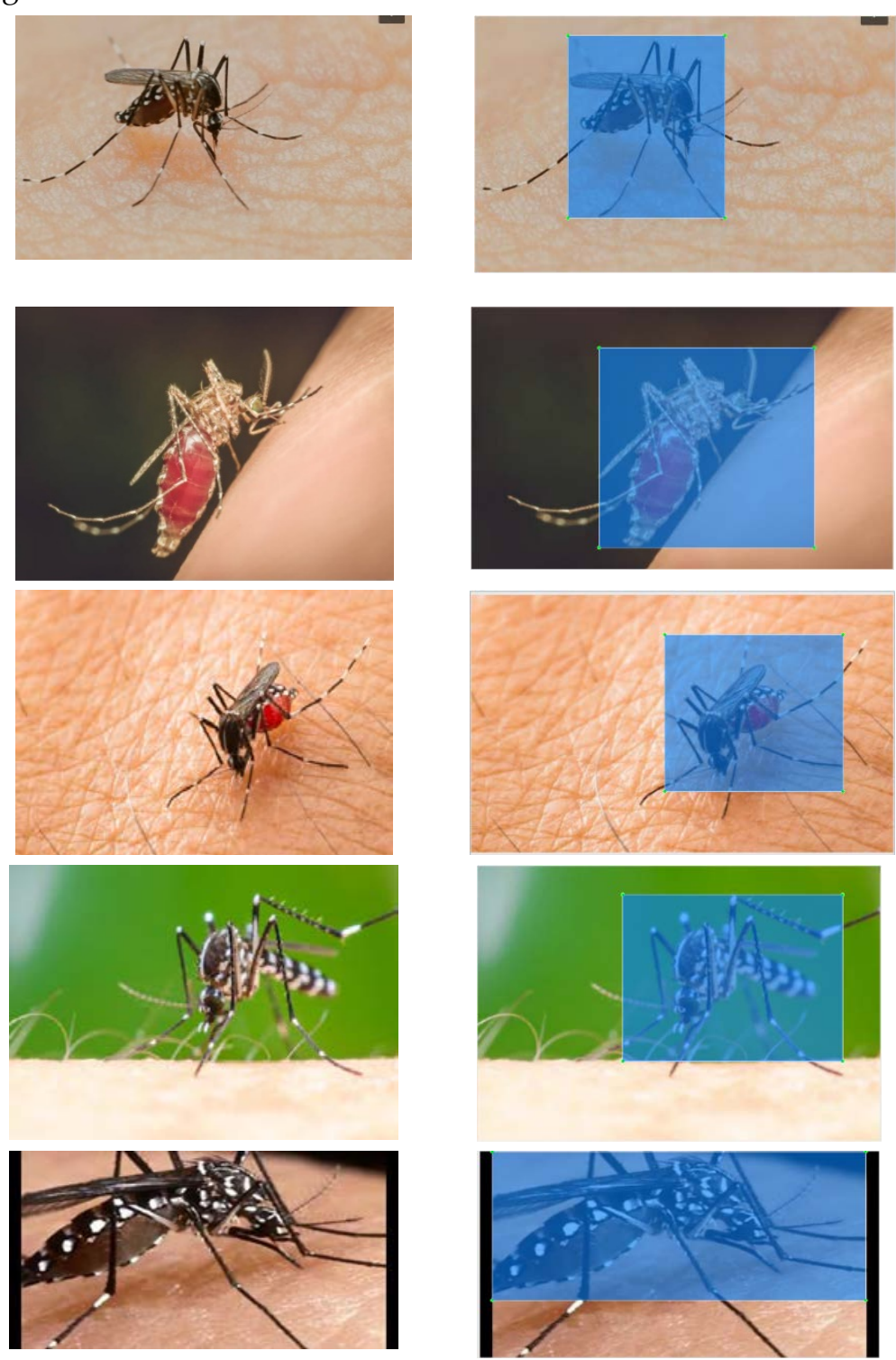

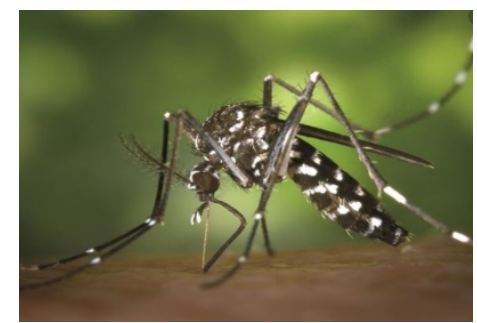

(a)

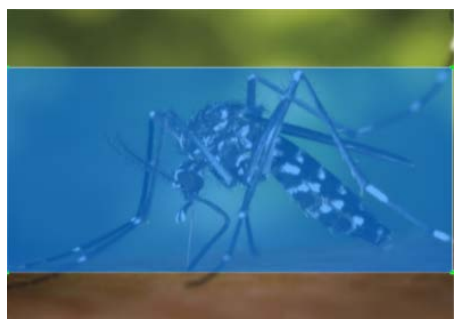

(b)

Figure 5. (a) Sample images and (b) respective annotated image 


\subsection{Data Processing}

Data processing is a method of transforming the data from a given process into a significantly more usable and preferred form by making it relevant and informative. After pre-processing phase, two steps are considered in the data process which generates comma separated value (CSV) and TFRecords as depicted in Figure 6.

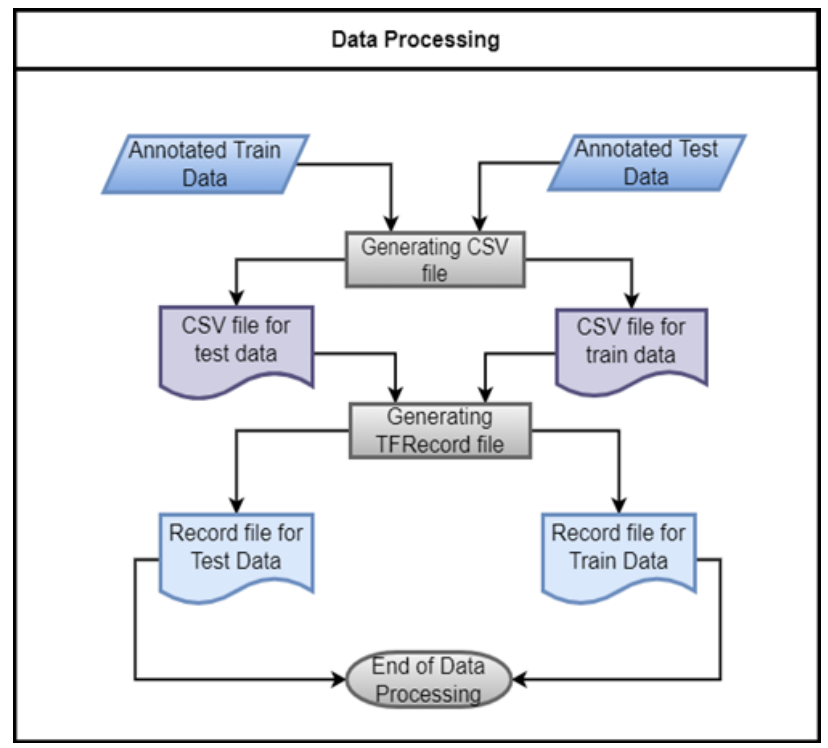

Figure 6. Workflow diagram for Data Processing

A CSV file is a delimited text file using a comma to distinguish values. Using this, a plain-text file has been generated in a tabular format for easy export and structured import of the data. A TFRecord file stores the data as a binary string sequence which means that the structure of the data needs to be specified before it is written to the file. With the labeled images, we need to build TFR records that are used for object detector training as an input data. In this way, a separate data file has been developed for storing a series of binary records.

\subsection{Process of training}

Lastly, before the training, we have to generate a label map and a training configuration file for the process. A text type document graph file, regarded as Label Map, is generated where the label map indicates if the object currently resides, and it is achieved by specifying the mapping of class names to class ID numbers. We have one 'dengue' mark so the ID number is 1 . Eventually, the training system for object detection needs to be designed. It is the last step before beginning the dengue detector classifier training, and the training pipeline for object detection must be configured. It determines which model should be used and which parameters are to be chosen for training. We also declared the number of evaluation steps of training in the configuration file, set the path of TFRecord test and train data files, number of evaluation data, label-map.pbtxt path. For object detection, modern meta-architectures use CNN. We have considered Faster R-CNN, R-FCN, and SSD metaarchitecture for dengue mosquito detection.

\subsection{Detected Data Evaluation}

After completing all the processes for the detection of dengue mosquitoes, the images of the different environmental situations are evaluated with the expected bounding boxes. For bounding box detection, Figure 7 shows all predicted regions in an image result where we identified dengue mosquitoes using the three selected models: Faster R-CNN, R-FCN, and SSD. In the observed bounding boxes, the percentages of detections are presented here and it varies in each image for the different models.

We are starting with the first dengue mosquito input image, followed by the output images of three different models. In Faster R-CNN, the detection percentage is $80 \%$, and the detection bounding box is one. However, there are three bounding detection boxes for R-FCNN and the detection 
percentage are $82 \%, 71 \%$, and $51 \%$, respectively, while there are two bounding boxes for the SSD model and the detection percentage is $55 \%$. Detection bounding box percentages for the Faster R$\mathrm{CNN}$ is $82 \%, \mathrm{R}-\mathrm{FCN}$ is $71 \%, 58 \%, 50 \%$ and $\mathrm{SSD}$ is $78 \%$. They are experienced for the second input image. Consequently, for the third input image, Faster R-CNN- $88 \%$ and $53 \%$, R-FCN- $63 \%$ and $69 \%$, and SSD- $72 \%, 70 \%$ and $55 \%$ are shown in the percentages of bounding box detection. For the fourth sample input image, detection bounding box percentages for Faster R-CNN is $69 \%$ and $64 \%$, R-FCN$57 \%$ and 54\%, and SSD- No detection. Similarly, analyzing for the last input image, detection bounding box percentages have been identified for Faster R-CNN- 75\% and 59\%, R-FCN- 91\%, 75\%, $65 \%, 62 \%$ and $59 \%$, and SSD- No detection.
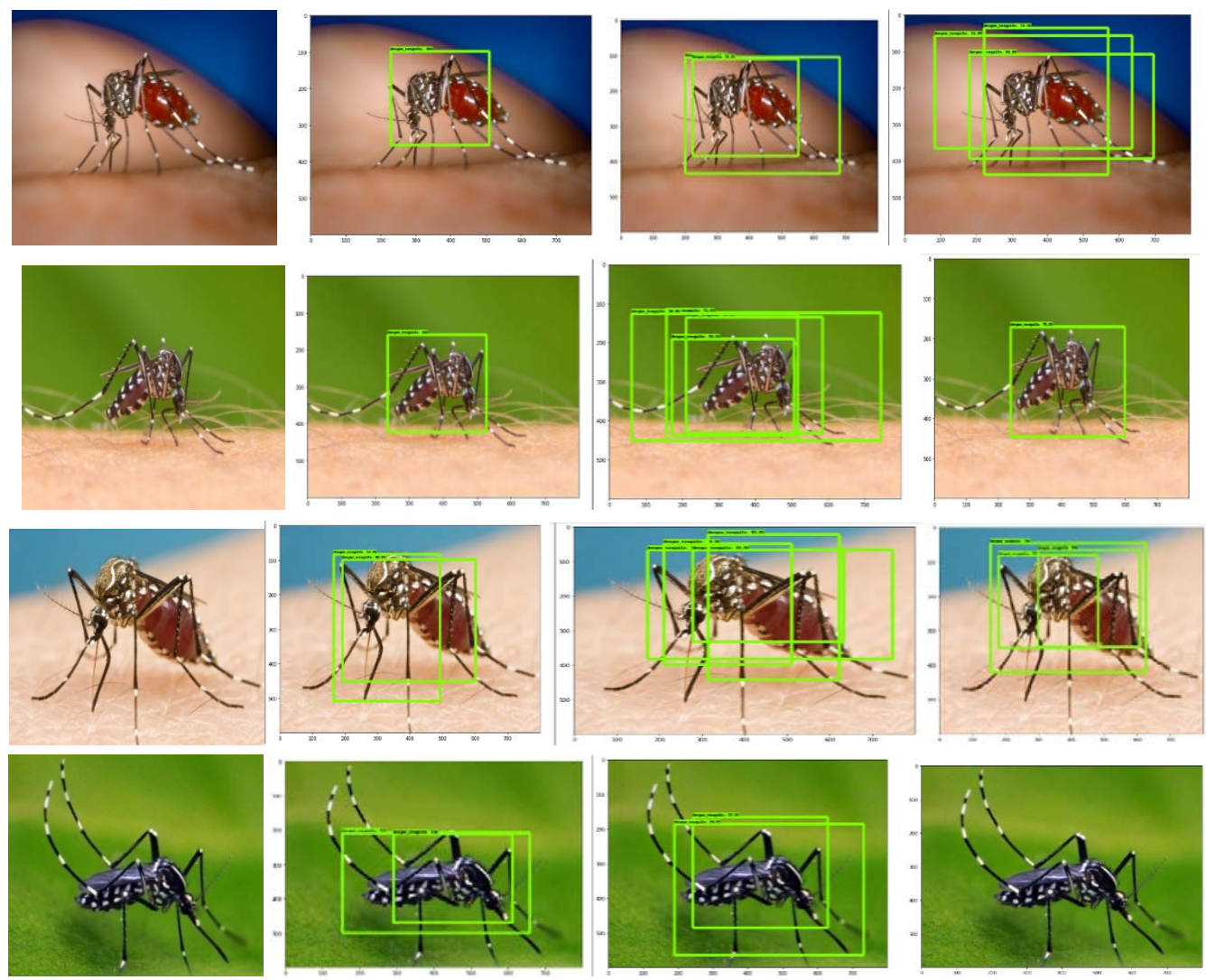

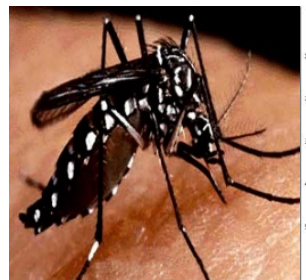

(a)

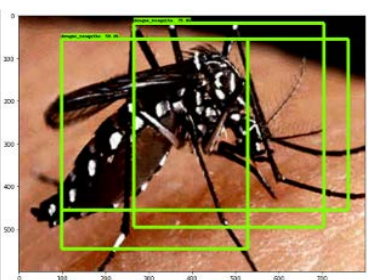

(b)

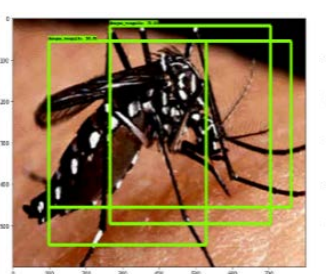

(c)

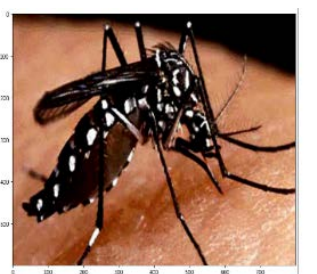

(d)

Figure 7. Dengue Mosquito Detection for different environments: (a) input image, (b) output of Faster R-CNN, (c) output of R-FCN, and (d) output of SSD

With the exception of the You Only Look Once (YOLO) [31] model, the Faster R-CNN, R-FCN, and SSD models do not predict single bounding boxes for the neural network here, that is why we have experienced the multiple bounding boxes for the three models.

\section{Experimental Analysis}

The experiment has been performed on secondary collected dataset of images from the different online resources and used python codes to resize all the raw images into 800x600 shapes. Due to the limited number of online images and difficulty to capture the images for the flying nature of the objects, forming a good dataset was challenging. For each image, the collected resized dengue mosquito images are acquired through a bounding box labelling process and an annotation file has been created containing bounding boxes. The collected image dataset is then divided into a training 
set and a test set for performance evaluation. The train and test datasets can be divided into $70 \%$ $30 \%, 80 \%-20 \%$, but due to the limited dataset, in the experimental evaluation we split them into $85 \%$ and $15 \%$. We have taken other insect images (as non-dengue) for detection to evaluate whether or not it is a dengue mosquito for an accurate depiction. The deep learning framework TensorFlow [32] version 1.x has been used to run the code for training on Google's Colab platform. We have used Intel Bay Trail M Quad-Core 3540 Processor, up to $2.66 \mathrm{GHz}$, for training and validation of dengue mosquito detection algorithms on a laptop.

The accuracy of the model has differed [33] due to the combination of the meta-architecture and the extractor. To select the optimal model, a comparison of these combinations is therefore required. By combining meta-architectures and feature extractors, we trained dengue mosquito detection models and compared each model's accuracy. The Faster R-CNN Inception V2, R-FCN RestNet 101, and SSD Mobilenet V2 models have been trained, compared, and analyzed.

In the performance evaluation, we have used the following statistical parameter: true positive (TP), true negative (TN), false positive (FP), false negative (FN), precision, and recall [34]. The true positive is the original dengue mosquito region that have been correctly identified. True negative represents a substantial part that does not pertain to the dengue mosquito region and is not performed to detect. False-positive represents an area that has been detected which is not present in the original dengue mosquito region. False negative refers to an area that is not identified and is also within the dengue mosquito's original region. After that, we recognize the coordinates of the original region area. So, considering the correctly detected area, we can determine the true positive. In the same way, we have evaluated other statistical parameters consequently. Precision or specificity represents the percentage of accurate positive predictions between several and it is calculated by Equation 1,

$$
\text { Precision }=\frac{\mathbf{T P}}{\mathbf{T P}+\mathbf{F P}}
$$

Percentage of total positive cases that classifiers can detect appropriately pertains to the recall, otherwise referred to as sensitivity, which is determined by Equation 2,

$$
\text { Recall }=\frac{\mathbf{T P}}{\mathbf{T P}+\mathbf{F N}}
$$

The accuracy is determined by Equation 3,

$$
\text { Accuracy }=\frac{\mathbf{T P}+\mathrm{TN}}{\mathbf{T P}+\mathbf{T N}+\mathbf{F P}+\mathbf{F N}}
$$

Table 1. Faster R-CNN model accuracy

\begin{tabular}{|l|l|l|l|l|l|l|}
\hline Proposed Model & $\begin{array}{l}\text { FP } \\
\mathbf{( \% )}\end{array}$ & FN (\%) & $\begin{array}{l}\text { Precision } \\
\mathbf{( \% )}\end{array}$ & $\begin{array}{l}\text { Recall } \\
\mathbf{( \% )}\end{array}$ & $\begin{array}{l}\text { Detection } \\
\text { Accuracy } \\
\text { according to TP, } \\
\text { TN, FP, FN (\%) }\end{array}$ & $\begin{array}{l}\text { Detection Accuracy for all } \\
\text { test images as a dengue } \\
\text { mosquito (\%) }\end{array}$ \\
\hline Faster R- CNN & 4.5 & 5.6 & 95.7 & 94.7 & 95.19 & 100 \\
\hline
\end{tabular}

Table 1 presents the performance of the detection which is evaluated with the four parameters: FP, FN, precision, and recall. Figure 8 demonstrates the efficiency comparison of this experiment's test set, which includes Faster R-CNN, R-FCN, and SSD methods. Throughout this experiment, we can conclude that the Faster R-CNN with Inception V2 model shows the better performance than others by exhibiting the highest detection accuracy of $95.19 \%$.

Figure 9 represents the erroneous results of non-dengue mosquito image detection. As depicted in Figure 9(b), the image for Faster R-CNN is not detected as a dengue mosquito image, which is an accurate result of this model. Figures 9(c) and 9(d), nevertheless, show the error result for R-FCN and SSD models, as the image is detected as a dengue mosquito of $77 \%$ and $55 \%$, respectively.

After comparing the output images for the three models, in particular, it has been analysed that SSD models show flawed results relative to other models. As shown in Figure 7, SSD failed to detect the last two images. Previous research in [35] stated that on small objects, SSD models show poor efficiency. Subsequently, all the images are detected for Faster R-CNN and R-FCN, but the percentages of every image varied due to the different training steps, as well as the period of completion of the training steps for each model. Faster R-CNN outperforms the R-CNN in terms of training steps. The R-FCN requires more time for each image to complete the training steps and detect 
with multiple detection bounding boxes compared to the Faster R-CNN model as shown in Table 2 . In this case, we can conclude that Faster R-CNN has performed a better detection accuracy than the R-FCN and SSD models.

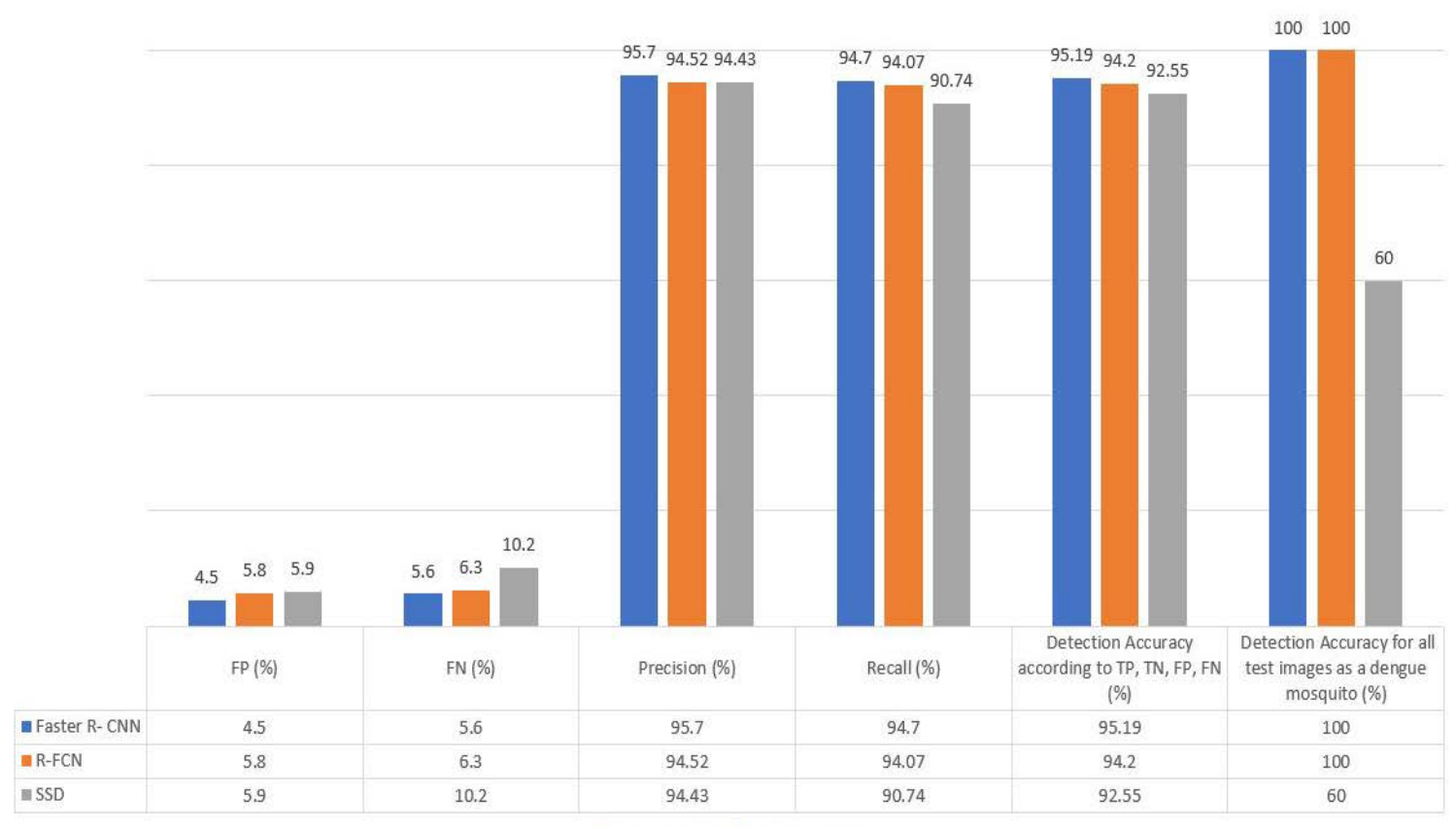

Figure 8. Statistical performance of the selected models

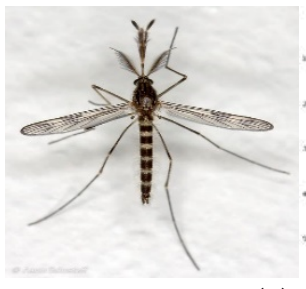

(a)

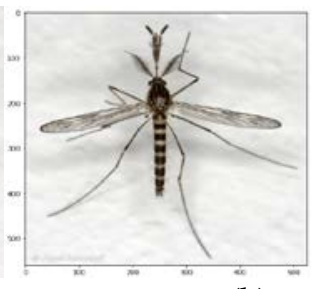

(b)

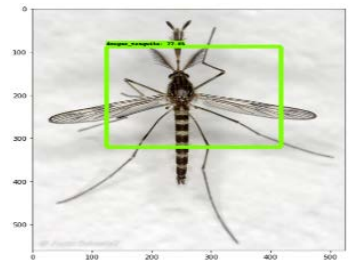

(c)

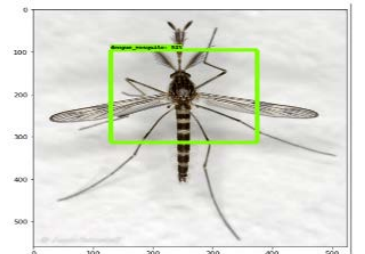

(d)

Figure 9. Experimental output for Non-Dengue mosquito image: (a) Input Image, (b) Output of Faster R-CNN, (c) Output of R-FCN, (d) Output of SSD

It is mentionable that due to the processor limitation, we fail to train the models for more than 10 hours. After 10 hours, the training steps were immediately terminated. If more steps are trained, the detection precision can be improved.

Table 2. Comparison of proposed model with other models

\begin{tabular}{|l|l|l|l|}
\hline Models & Training step & Training Duration & $\begin{array}{l}\text { Batch } \\
\text { Size }\end{array}$ \\
\hline R-FCN_Resnet101 & 400 & 10 hours & 8 \\
\hline SSD_MobilenetV2 & 8000 & 10 hours & 12 \\
\hline $\begin{array}{l}\text { Proposed Faster_R- } \\
\text { CNN_InceptionV2 }\end{array}$ & 800 & 10 hours & 12 \\
\hline
\end{tabular}

\section{Conclusion}

In this paper, we have used deep learning algorithm and image processing techniques to detect dengue mosquitos from the images obtained from various relevant sources. To identify dengue mosquitoes, we use the Faster R-CNN with InceptionV2, R-FCN with ResNet101, and SSD with MobilenetV2 models. We train these three models and apply them for real-time recognition by using the training data. The experimental results show that the Faster R-CNN with the InceptionV2 model can reliably identify whether the dengue mosquito exists or not in an image compared to the other two proposed models. In addition, the method of dengue mosquito recognition from the image is very precise and accurate with this deep learning-based approach, as opposed to other mosquito 
species. In the future, work will be conducted to apply the method in dynamic situations in real-time environments.

\section{Acknowledgement}

This research is funded by Woosong University Academic Research in 2021.

\section{References}

[1] Ayan K. Biswas, Nahian A. Siddique, Bing B. Tian, Enoch Wong, Kevin A. Caillouët et al., “Design of a FiberOptic Sensing Mosquito Trap", IEEE Sensors Journal, Online ISSN: 1530-437X, E- ISSN: 1558-1748, pp. 44234431, Vol. 13, No. 11, November 2013, Published by IEEE, DOI: 10.1109/JSEN.2013.227427, Available: https://ieeexplore.ieee.org/document/6566019.

[2] Mona Minakshi, Pratool Bharti and Sriram Chellappan, "Leveraging Smart-Phone Cameras and Image Processing Techniques to Classify Mosquito Species", in Proceedings of the 15th EAI International Conference on Mobile and Ubiquitous Systems: Computing, Networking and Services 2018 (MobiQuitous '18), November 2018, Published by Association for Computing Machinery, New York, NY, USA, pp. 77-86. DOI: 10.1145/3286978.3286998, Available: https://dl.acm.org/doi/abs/10.1145/3286978.3286998.

[3] Samir Bhatt, Peter W. Gething, Oliver J. Brady, Jane P. Messina, Andrew W. Farlow et al., "The global distribution and burden of dengue", Nature, PMID: 23563266, pp. 504-507, Vol. 496, No. 7446, 25th April 2013, DOI: 10.1038/nature12060, Available: https://pubmed.ncbi.nlm.nih.gov/23563266/.

[4] Suk-Ju Hong, Sang-Yeon Kim, Eungchan Kim, Chang-Hyup Lee, Jung-Sup Lee et al., "Moth Detection from Pheromone Trap Images Using Deep Learning Object Detectors", Agriculture, p. 170, Vol. 10, No. 5, 14th May 2020, Published by MDPI, DOI: 10.3390/agriculture10050170, Available: https://www.mdpi.com/2077$\underline{0472 / 10 / 5 / 170 .}$.

[5] Md. Nazmus Sabab, Mohammad Abidur Rahman Chowdhury, S. M. Mahsanul Islam Nirjhor and Jia Uddin, "Bangla Speech Recognition Using 1D-CNN and LSTM with Different Dimension Reduction Techniques", Emerging Technologies in Computing. iCETiC 2020. Lecture Notes of the Institute for Computer Sciences, Social Informatics and Telecommunications Engineering, Print ISBN: 978-3-030-60035-8, Online ISBN: 978-3-030-60036-5, pp. 158-169, Vol. 332, 29th September 2020, Published by Springer, Cham, DOI: 10.1007/978-3-030-60036-5_11, Available at: https://link.springer.com/chapter/10.1007/978-3-030-60036-5 11.

[6] Zhong-Qiu Zhao, Peng Zheng, Shou-Tao Xu and Xindong Wu, “Object Detection with Deep Learning: A Review", IEEE Transactions on Neural Networks and Learning Systems, Print ISSN: 2162-237X, Electronic ISSN: 2162-2388, pp. 3212-3232, Vol. 30, No. 11, November 2019, Published by IEEE, DOI: 10.1109/TNNLS.2018.2876865, Available: https://ieeexplore.ieee.org/abstract/document/8627998.

[7] Shaoqing Ren, Kaiming He, Ross Girshick and Jian Sun, "Faster R-CNN: Towards Real-Time Object Detection with Region Proposal Networks", IEEE Transactions on Pattern Analysis and Machine Intelligence, Print ISSN: 0162-8828, Electronic ISSN: 1939-3539, pp. 1137-1149, Vol. 39, No. 6, 1st June 2017, Published by IEEE, DOI: 10.1109/tpami.2016.2577031, Available: https://ieeexplore.ieee.org/abstract/document/7485869.

[8] Jifeng Dai, Yi Li, Kaiming He and Jian Sun, "R-FCN: Object detection via region-based fully convolutional networks", in Proceedings of the 30th International Conference on Neural Information Processing Systems (NIPS'16), December 2016, Red Hook, NY, USA, Online ISBN: 9781510838819, pp. 379-387, Published by Curran Associates Inc., Available: https://dl.acm.org/doi/10.5555/3157096.3157139.

[9] Wei Liu, Dragomir Anguelov, Dumitru Erhan, Christian Szegedy, Scott Reed et al., "SSD: Single Shot MultiBox Detector", in Lecture Notes in Computer Science, Computer Vision - ECCV, Vol 9905, Online ISBN: 978-3-319-46448-0, Print ISBN: 978-3-319-46447-3, DOI: 10.1007/978-3-319-46448-0_2, pp. 21-37, 2016, Published by Springer-Cham, Available: https://link.springer.com/chapter/10.1007/978-3-319-46448-0 2.

[10] Derry Alamsyah and Muhammad Fachrurrozi, "Faster R-CNN with Inception V2 for Fingertip Detection in Homogenous Background Image", Journal of Physics: Conference Series, Vol. 1196, 2018, DOI: 10.1088/17426596/1196/1/012017, Available: https://iopscience.iop.org/article/10.1088/1742-6596/1196/1/012017.

[11] Kaiming He, Xiangyu Zhang, Shaoqing Ren and Jian Sun, "Deep residual learning for image recognition", in Proceedings of the IEEE conference on computer vision and pattern recognition (CVPR), 27-30 June 2016, Online ISBN: 978-1-4673-8852-8, E-ISBN: 978-1-4673-8851-1, DOI: 10.1109/CVPR.2016.90, pp. 770-778, Published by IEEE, Available: https://ieeexplore.ieee.org/document/7780459.

[12] Mark Sandler, Andrew Howard, Menglong Zhu, Andrey Zhmoginov and Liang-Chieh Chen, "MobilenetV2: Inverted residuals and linear bottlenecks", in Proceedings of the IEEE Conference on Computer Vision and Pattern Recognition (CVPR), 18-23 June 2018, Online ISBN: 978-1-5386-6421-6, E-ISBN: 978-1-53866420-9, DOI: 10.1109/CVPR.2018.00474, pp. 4510-4520, Published by IEEE, Available: https://ieeexplore.ieee.org/document/8578572. 
[13] N. Dalal and B. Triggs, "Histograms of Oriented Gradients for Human Detection", in Proceedings of the IEEE Computer Society Conference on Computer Vision and Pattern Recognition (CVPR'05), 20-25 June 2005, Online ISBN: 0-7695-2372-2, DOI: 10.1109/CVPR.2005.177, pp. 886-893, vol. 1, Published by IEEE, Available: https://ieeexplore.ieee.org/abstract/document/1467360.

[14] P. Viola and M. Jones, "Rapid object detection using a boosted cascade of simple features", in Proceedings of the 2001 IEEE Computer Society Conference on Computer Vision and Pattern Recognition (CVPR 2001), 8-14 Dec. 2001, Online ISBN: 0-7695-1272-0, DOI: 10.1109/CVPR.2001.990517, pp. 1-1, Published by IEEE, Available: https://ieeexplore.ieee.org/abstract/document/990517.

[15] Johan A.K. Suykens, Jos De Brabanter, Lukas and Joos Vandewalle, "Weighted least squares support vector machines: robustness and sparse approximation", Neurocomputing, pp. 85-105, Vol. 48, No. 1-4, 29th August 2002, Published by Elsevier, DOI: 10.1016/s0925-2312(01)00644-0, Available: https://www.sciencedirect.com/science/article/abs/pii/S0925231201006440.

[16] Warren S. McCulloch and Walter Pitts, "A logical calculus of the ideas immanent in nervous activity", Bulletin of Mathematical Biophysics, pp. 115-133, Vol. 5, December 1943, Published by Springer, DOI: 10.1007/BF02478259, Available: https://link.springer.com/article/10.1007/BF02478259.

[17] Partha Narayan Chowdhury, Tonmoy Chandra Ray and Jia Uddin, "A Vehicle Detection Technique for Traffic Management using Image Processing", in 2018 International Conference on Computer, Communication, Chemical, Material and Electronic Engineering (IC4ME2), 20 September 2018, Online ISBN:978-1-5386-4775-2, E-ISBN:978-1-5386-4776-9, doi: 10.1109/IC4ME2.2018.8465599, pp. 1-4, Published by IEEE, Available: https://ieeexplore.ieee.org/abstract/document/8465599.

[18] Christian Szegedy, Wei Liu, Yangqing Jia, Pierre Sermanet, Scott Reed et al., "Going deeper with convolutions", in Proceedings of the IEEE Conference on Computer Vision and Pattern Recognition (CVPR), 7-12 June 2015, ISBN: 978-1-4673-6963-3, E-ISBN: 978-1-4673-6964-0, DOI: 10.1109/CVPR.2015.7298594, pp. 1-9, Published by IEEE, Available: https://ieeexplore.ieee.org/document/7298594.

[19] Alex Krizhevsky, Ilya Sutskever, and Geoffrey E. Hinton, "ImageNet classification with deep convolutional neural networks", Communications of the ACM, Online ISSN: 0001-0782, pp. 84-90, 2017, Vol. 60, No. 6, June 2017, DOI: 10.1145/3065386, Available: https://dl.acm.org/doi/10.1145/3065386.

[20] Daniel Motta, Alex Á. B. Santos, Ingrid Winkler, Bruna A. S. Machado, Daniel A. D. Imperial Pereira et al., "Application of convolutional neural networks for classification of adult mosquitoes in the field", PLOS ONE, Vol. 14, No. 1, p. e0210829, 14th January 2019, DOI: 10.1371/journal.pone.0210829, Available: https://journals.plos.org/plosone/article?id=10.1371/journal.pone.0210829.

[21] Yuanhong Zhong, Junyuan Gao, Qilun Lei and Yao Zhou, "A Vision-Based Counting and Recognition System for Flying Insects in Intelligent Agriculture", Sensors, Vol. 18, No. 5, p. 1489, May 2018, Published by MDPI, DOI: 10.3390/s18051489, Available: https://www.mdpi.com/1424-8220/18/5/1489.

[22] Hulya Yalcin, "Vision based automatic inspection of insects in pheromone traps", in Proceedings of the Fourth International Conference on Agro-Geoinformatics (Agro-geoinformatics), 20-24 July 2015, ISBN: 978-1-4673-73180, E-ISBN: 978-1-4673-8087-4, DOI: 10.1109/Agro-Geoinformatics.2015.7248113, pp. 333-338, Published by IEEE, Available: https://ieeexplore.ieee.org/abstract/document/7248113.

[23] Kazushige Okayasu, Kota Yoshida, Masataka Fuchida and Akio Nakamura, "Vision-Based Classification of Mosquito Species: Comparison of Conventional and Deep Learning Methods", Applied Sciences, Vol. 9, No. 18, p. 3935, 19th September 2019, Published by MDPI, DOI: 10.3390/app9183935, Available: https://www.mdpi.com/2076-3417/9/18/3935.

[24] Masataka Fuchida, Thejus Pathmakumar, Rajesh E. Mohan, Ning Tan and Akio Nakamura, "Vision-Based Perception and Classification of Mosquitoes Using Support Vector Machine", Applied Sciences, Vol. 7, No. 1, p. 51, 5th January 2017, Published by MDPI, DOI: 10.3390/app7010051, Available: https://www.mdpi.com/2076-3417/7/1/51.

[25] Anna Monica M. De Los Reyes, Anna Camille A. Reyes, Jumelyn L. Torres, Dionis A. Padilla and Jocelyn Villaverde, "Detection of Aedes Aegypti mosquito by digital image processing techniques and support vector machine", in Proceedings of the IEEE Region 10 Conference (TENCON), 22-25 November 2016, Online ISBN:978-1-5090-2598-5, E-ISBN: 978-1-5090-2597-8, DOI: 10.1109/TENCON.2016.7848448, pp. 2342-2345, Published by IEEE, Available: https://ieeexplore.ieee.org/document/7848448.

[26] Treesukon Treebupachatsakul and Suvit Poomrittigul, "Bacteria Classification using Image Processing and Deep learning", in Proceedings of the 34th International Technical Conference on Circuits/Systems, Computers and Communications (ITC-CSCC), 23-26 June 2019, Online ISBN:978-1-7281-3272-3, E-ISBN: 978-1-7281-3271-6, DOI: 10.1109/ITC-CSCC.2019.8793320, pp. 1-3, Published by IEEE, Available: https://ieeexplore.ieee.org/document/8793320.

[27] Mona Minakshi, Pratool Bharti and Sriram Chellappan, "Identifying mosquito species using smart-phone cameras", in Proceedings of the European Conference on Networks and Communications (EuCNC), 12-15 June 2017, 
Online ISBN: 978-1-5386-3874-3, E-ISBN: 978-1-5386-3873-6, DOI: 10.1109/EuCNC.2017.7980646, pp. 1-6, Published by IEEE, Available: https://ieeexplore.ieee.org/abstract/document/7980646.

[28] Maxime Martineau, Donatello Conte, Romain Raveaux, Ingrid Arnault, Damien Munier et al., "A survey on image-based insect classification", Pattern Recognition, pp. 273-284, Vol. 65, 23rd December 2016, Published by Elsevier, DOI: 10.1016/j.patcog.2016.12.020.

[29] Jerrid Matthews, Rajan Kulkarni, George Whitesides, Majid Sarrafzadeh, Mario Gerla et al., "A light-weight solution for real-time dengue detection using mobile phones", in Proceedings of the IEEE Computer Society MOBICASE 2009, San Diego, CA, United States, October 2009, Available: https://citeseerx.ist.psu.edu/viewdoc/download?doi=10.1.1.556.8590.

[30] Duc N. Pham, Tarique Aziz, Ali Kohan, Syahrul Nellis, Juraina binti Abd. Jamil et al., "How to Efficiently Predict Dengue Incidence in Kuala Lumpur", in Proceedings of the Fourth International Conference on Advances in Computing, Communication \& Automation (ICACCA), 26-28 Oct. 2018, Online ISBN:978-1-5386-7168-9, EISBN: 978-1-5386-7167-2, DOI: 10.1109/ICACCAF.2018.8776790, pp. 1-6, Published by IEEE, Available: https://ieeexplore.ieee.org/abstract/document/8776790.

[31] Joseph Redmon, Santosh Divvala, Ross Girshick, Ali Farhadi, "You only look once: Unified, real-time object detection", in Proceedings of the IEEE Conference on Computer Vision and Pattern Recognition (CVPR), 27-30 June 2016, Online ISBN:978-1-4673-8852-8, E-ISBN: 978-1-4673-8851-1, DOI: 10.1109/CVPR.2016.91, pp. 779-788, Published by IEEE, Available: https://ieeexplore.ieee.org/document/7780460.

[32] Martín Abadi, Paul Barham, Jianmin Chen, Zhifeng Chen, Andy Davis et al., "TensorFlow: a system for large-scale machine learning", in Proceedings of the 12th USENIX conference on Operating Systems Design and Implementation (OSDI'16), 2016, Savannah, GA, USA, Online ISBN 9781931971331, pp. 265-283, Published by USENIX Association, Available: https://dl.acm.org/doi/10.5555/3026877.3026899.

[33] Jonathan Huang, Vivek Rathod, Chen Sun, Menglong Zhu, Anoop Korattikara et al., "Speed/accuracy tradeoffs for modern convolutional object detectors", in Proceedings of the IEEE conference on computer vision and pattern recognition (CVPR), 21-26 July 2017, Online ISBN- 978-1-5386-0458-8, E-ISBN: 978-1-5386-0457-1, DOI: 10.1109/CVPR.2017.351, pp. 7310-7311, Available: https://ieeexplore.ieee.org/document/8099834.

[34] Mahe Zabin, Ho-Jin Choi, Jia Uddin, Md. Hasan Furhad and Abu.Barkat Ullah, "Industrial Fault Diagnosis using Hilbert Transform and Texture Features", in Proceedings of the 2021 IEEE International Conference on Big Data and Smart Computing (BigComp), 10 March 2021, Online ISBN- ISBN:978-1-7281-8925-3, E-ISBN- 978-17281-8924-6, doi: 10.1109/BigComp51126.2021.00031, pp. 121-128, Published by IEEE, Available: https://ieeexplore.ieee.org/abstract/document/9373237.

[35] Nikhil Yadav and Utkarsh Binay, "Comparative study of object detection algorithms", International Research Journal of Engineering and Technology (IRJET), E-ISSN: 2395-0056, P-ISSN: 2395-0072, pp. 586-591, Vol. 04, Issue: 11, November 2017, Available: https://www.irjet.net/archives/V4/i11/IRJET-V4I11103.pdf.

(C) 2021 by the author(s). Published by Annals of Emerging Technologies in Computing (AETiC), under the terms and conditions of the Creative Commons Attribution (CC BY) license which can be accessed at http://creativecommons.org/licenses/by/4.0. 